\title{
A study of the development of global solar activity in the 23 rd solar cycle based on radio observations with the Nobeyama radio heliograph
}

\section{Latitude distribution of the active and dark regions}

\author{
G. B. Gelfreikh ${ }^{1}$, V. I. Makarov ${ }^{1}$, A. G. Tlatov ${ }^{1}$, A. Riehokainen ${ }^{2}$, and K. Shibasaki ${ }^{3}$ \\ 1 Pulkovo Astronomical Observatory, 196140 Saint Petersburg, Russia \\ 2 Tuorla Observatory, 21500 Piikkiö, Finland \\ 3 Nobeyama Radio Observatory, Minamisaku, Nagano 384-13, Japan
}

Received 11 October 2001 / Accepted 15 April 2002

\begin{abstract}
Daily radio maps of the Sun at the wavelength of $1.76 \mathrm{~cm}$ were used to analyze the parameters of solar activity at all heliographic latitudes for the period 1992-2001. As a criterion of the level of solar activity, we analyzed the area/number of regions with an excess of brightness above a certain fixed level as well as regions with brightness below a certain level. The distribution of such "bright" and "dark" regions with heliographic latitude as function of time was found. Special attention was paid to the high latitude polar regions where the ways of analyzing solar activity are rather limited and have no generally accepted methods. The results are compared with some other indices of high latitude solar activity, such as polar faculae and magnetic field measurements. They appear to be in general agreement with the radio observations. The advantage of using radio observations is a more homogeneous database and a stable method of analysis.
\end{abstract}

Key words. Sun: activity - Sun: sunspots - Sun: magnetic fields - Sun: radio radiation

\section{Introduction}

The usual approach to studying solar activity, up to the 1980s, has been to investigate the sunspot cycle. Wolf's numbers and sunspot areas were considered to be the main characteristics of the solar activity, partly due to the long time span of observations. Other important observational data, such as radio flux at $10.7 \mathrm{~cm}$, magnetic field strength, brightness of the solar corona, etc., have also been obtained for about 50 years. Because of the available data, solar activity investigations have been limited almost exclusively to low or middle latitudes. However, there is much evidence showing that the duration of the solar cycle is longer than 11 years. The most important evidence is the following: discovery of torsional oscilations (Howard \& LaBonte 1980); study of the long time variations of the geomagnetic activity and of the solar wind (Legrand \& Simon 1983); study of the solar coronal variations during a few cycles (Leroy \& Noens 1983; Wilson et al. 1988); study of the topology and the polarity changes of the large-scale magnetic field (Makarov \& Sivaraman 1989); study of the polar solar activity (Makarov \& Makarova 1996)

Send offprint requests to: A. Riehokainen, e-mail: alrieho@astro.utu.fi and study of the solar activity in radio waves (Gelfreikh et al. 2000).

It has become evident that a realistic theory explaining the physical nature of the solar activity and its cycles needs an observational data base which includes all heliographic latitudes. Nevertheless, while low and middle latitude manifestations of the solar active regions have been studied in details for many decades (or even centuries), analysis of the polar zones has been very limited. This is due both to technical problems (low contrast and size of the polar faculae, etc.) and a lack of recognition of the great significance of polar structures for the theory of the cycle. Detection of a correlation between the level of activity in the equatorial regions and the preceding activity at the high solar latitudes (Makarov \& Makarova 1996) was a breakthrough in the problem and has led to a significant growth of attention to observations of all types of structures at high heliospheric latitudes and to investigations of the solar cycle as an essentially global process (Makarov \& Sivaraman 1989). It has been shown that the large-scale magnetic field cycle is, on the average, 5.5 year ahead of the 11-year sunspot cycle (Makarov et al. 2001). It was also shown that in the polar zones of the Sun the area covered by unipolar magnetic fields during minimum sunspot activity has risen by a factor of 2 from 
1878-1996. This means that the behaviour of the geomagnetic index, and consequently the magnetic flux from the Sun, may be explained by an increase in the polar cap area with a roughly constant magnetic field in this period (Makarov et al. 2002, in press).

However, while low altitude active regions of the Sun are easy to observe even with moderate technical means, we have only rather limited data on high latitude solar activity. Different types of activity have been difficult to analyse using homogeneous methods. In this respect the data bank of the Nobeyama Radio Observatory obtained with the radio heliograph at the wavelength of $1.76 \mathrm{~cm}$ is of special interest. The regular (eight hours per day) observations are available since July 1992, with radio maps of the whole solar disk with a two-dimensional resolution of 10-15 arcsec (Nakajima et al. 1994). So, at present we can analyze both the high latitude parameters of the $23 \mathrm{rd}$ cycle, which are seen at the polar regions near the minimum of the previous 22nd cycle, and the development of activity near its maximum in 2001.

The main goal of this paper is to obtain the latitudetime distribution of the different ("bright" and "dark") radio regions and to compare it with the results obtained from the optical observations.

Another goal of this paper is to develop some methods of analysis of the solar activity as a global process, based on the Nobeyama data for the period 1992-2001. The effectiveness of Nobeyama data in studying the solar activity at all heliographic latitudes has been shown earlier, in principle (Gelfreikh 1999; Shibasaki 1999). Here we continue the investigation and especially illustrate the development of the polar activity in the polar zones at the border of the two cycles. We also analyze "dark" radio regions, mostly connected with the filaments, which also reflect some special features in the structure of the general magnetic field of the Sun.

An analysis of solar differential rotation and its variation with the phase of the solar activity was also possible. These results will be presented in a companion paper.

\section{Data analysis}

The daily (one per day) maps of the radio intensity in FITS format were used to study the development of the solar activity in the period July 1992 - June 2001. The Nobeyama radio heliograph provides a two-dimensional resolution of about 10 arcsec. The radio maps had a pixels size of 4.9 by $4.9 \operatorname{arcsec}$ (so, the solar radius was about 200 pixels). A heliographic coordinate grid was superposed on the radio map. The brightness of the quiet Sun can be found from the maximum of the pixel number distribution as a function of temperature over the solar disk. One example of such a distribution is given in Fig. 1.

Between 1992-1999 the temperature of the quiet Sun, according to the Nobeyama radio heliograph observations, did not change considerably, remaining in the range 10260-10300 K. In the year 2000 the temperature increased to $10370 \mathrm{~K}$ and in 2001 it increased to $10420 \mathrm{~K}$.

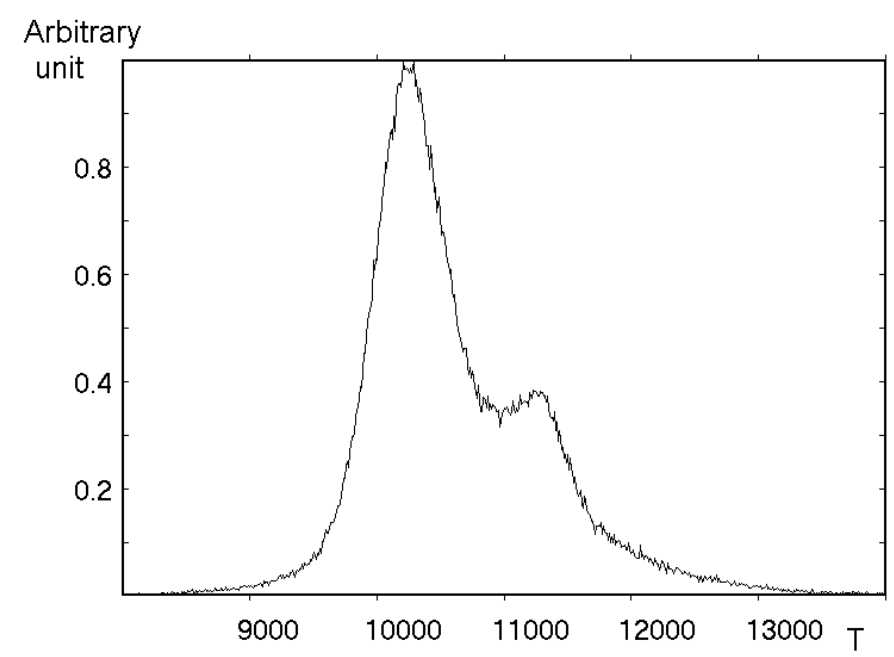

Fig. 1. A typical example of the distribution of the brightness of the radio map pixels over the whole solar disk. The number of the pixels is given in arbitrary units. The largest maximum corresponds to the quiet Sun level, the smaller one to active regions.

The regions exceeding a certain level of brightness were included in the analysis. A similar procedure was used for dark regions. We have chosen in our study $T_{\mathrm{b}}>11800 \mathrm{~K}$ as the optimal level for the "bright" regions and $T_{\mathrm{b}}<$ $9700 \mathrm{~K}$ for the "dark" ones. As is shown in Fig. 1, the regions in these temperatures ranges have less than 10 percent of the total number of pixels. Comparisons with the optical observations show a good correspondence with boundaries of the calcium flocculi (for $T_{\mathrm{b}}>11800 \mathrm{~K}$ ) and $\mathrm{H}$-alpha filaments (for $T_{\mathrm{b}}<9700 \mathrm{~K}$ ). A small change in either value would not change any of our conclusions appreciably.

For both the bright and the dark regions we calculated their areas with correction for projection effects in units of a millionth of the solar hemisphere, the coordinates of the effective center and the extent in heliographic latitude and longitude. The mean brightness for each region was also found. In the statistical study only the regions with an area exceeding 10 units were used for latitudes higher than $50^{\circ}$, and regions exceeding 200 units for latitudes below $50^{\circ}$. These different selection criteria for high and low latitude areas were adopted in order to simplify comparisons with previous optical observations of polar and low latitude faculae (Makarov \& Makarova 1996).

To exclude limb effects we did not consider regions beyond 0.98 of the solar radius.

For the period 1992-2001 the number of bright regions was found to be 86700,37400 of them at high latitudes exceeding $50^{\circ}$. The number of dark regions was about 83800 , including 13500 at high latitudes. 


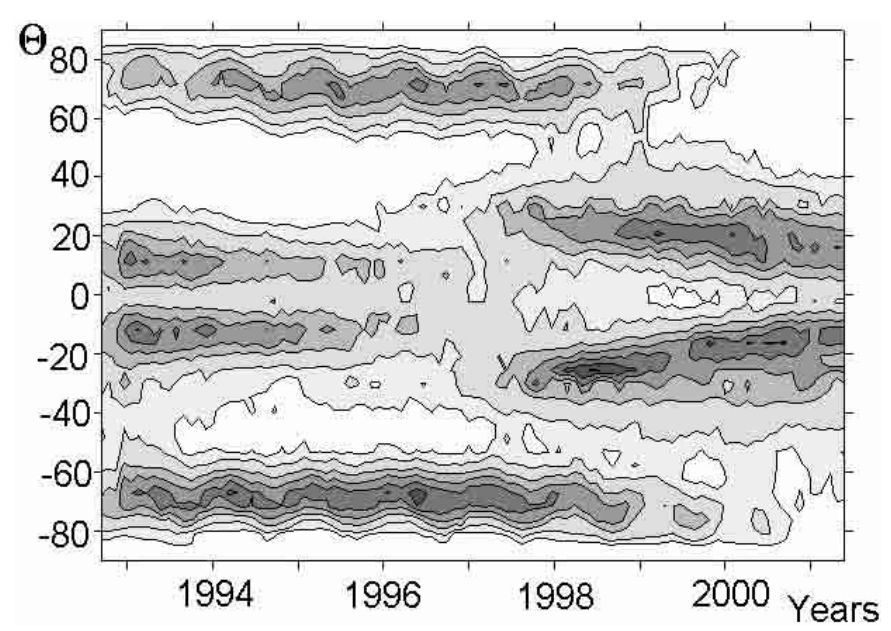

Fig. 2. The latitude-time distribution of the number bright regions for the radio observations at $1.76 \mathrm{~cm}$ with $T_{\mathrm{b}}>11800 \mathrm{~K}$.

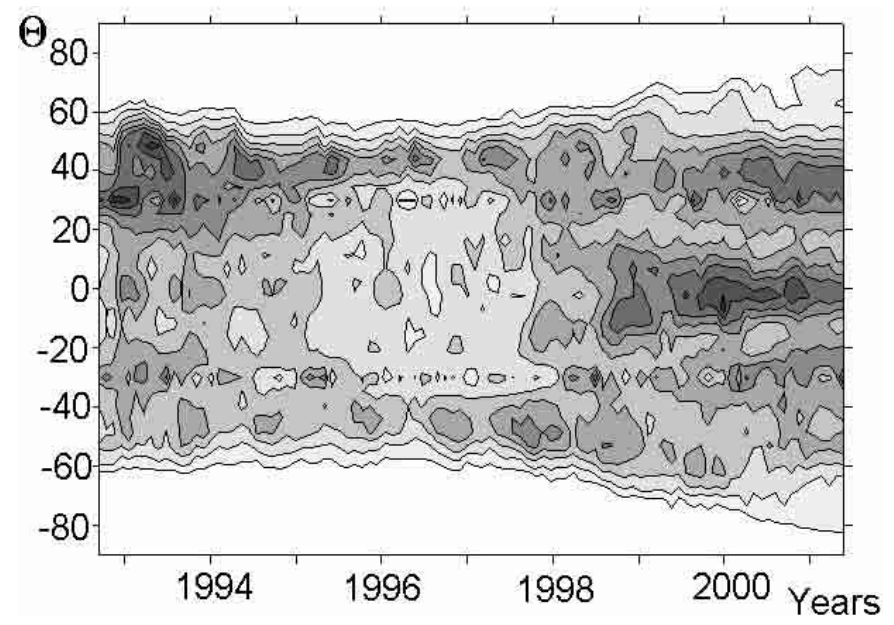

Fig. 3. The latitude-time distribution of the number dark regions for the radio observations at $1.76 \mathrm{~cm}$ with $T_{\mathrm{b}}<9700 \mathrm{~K}$.

\section{Results}

\subsection{The latitude distribution of the number of bright and dark regions}

During the period 1992-2001 there were two latitude belts in each solar hemisphere where an increase the radio brightness was registered. One region, around $40^{\circ}$ from the equator was due to increased emission of the active regions. The second belt was situated in the high solar latitudes (see Fig. 2). The distribution is very similar to the latitude-time distribution of the brightness of the corona in FeXIV $5303 \AA$ (Leroy \& Noens 1983).

The dark radio regions were found to have a maximum around the latitude of about 40 degrees (see Fig. 3). The distribution looks similar to the distribution of the filaments. One can see both the low latitude belts (around 20 degrees) and the high latitude belts (above 40 degrees) drifting towards the poles.

Figures 2 and 3 show the latitude-time variations of the bright and the dark regions averaged over monthly intervals for the period of 1992-2001. For the bright regions

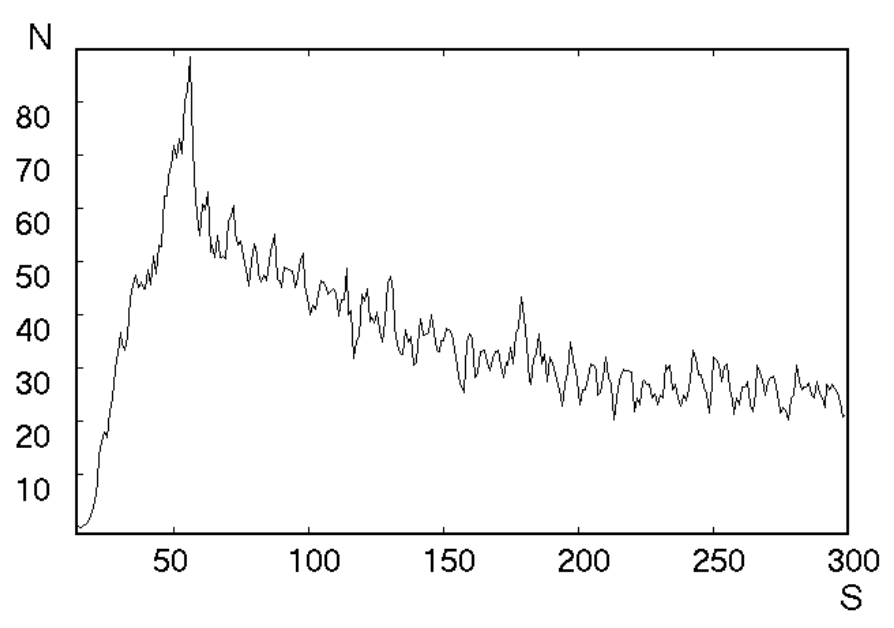

Fig. 4. The area distribution of bright $\left(T_{\mathrm{b}}>11800 \mathrm{~K}\right)$ regions at latitudes exceeding $50^{\circ}$.

we see the normal well-defined butterfly diagram at low latitudes. The development of solar activity at high latitudes is also clearly seen. Dark areas are practically absent at high latitudes. Their drift to higher latitudes after 1998 reflects the known effect (Makarov \& Sivaraman 1989) of the shift of the solar magnetic field neutral lines at this phase of the solar activity (see also Fig. 8).

\subsection{The area distribution of the bright and the dark regions}

We begin with an analysis of the distribution of the areas and the temperatures of bright $\left(T_{\mathrm{b}}>11800 \mathrm{~K}\right)$ regions at the latitudes exceeding $50^{\circ}$ (Fig. 4). The area distribution has a maximum at about 55 millionths of the hemisphere area. It may be connected with the spatial resolution of the Nobeyama radioheliograph. In principle, this distribution is typical for any kind of bright structure that can be observed in the solar atmosphere. The number of these regions decreases with increasing area. At latitudes less than 40 degrees we have studied the regions with areas less than 200 millionths of the solar hemisphere. In this case the number of regions decreases smoothly with increasing area. The same behaviour was established for the dark regions, too.

\subsection{The temperature distribution}

The average temperatures of the bright and the dark regions were obtained. This gives us the possibility to study the temperatures characterizing the high and the low latitude regions. For example, the brightness distribution shows a sharp maximum at $11900 \mathrm{~K}$ which is an artifact following from the chosen cut-off level. A second, smoother maximum for high latitude regions is seen at approximately $12350 \mathrm{~K}$ (Fig. 5). For higher brightness, the number of regions drops quickly. Similar distributions for bright and for dark $\left(T_{\mathrm{b}}<9700 \mathrm{~K}\right)$ regions at low latitudes show no maximum, only a smooth decrease. It is 


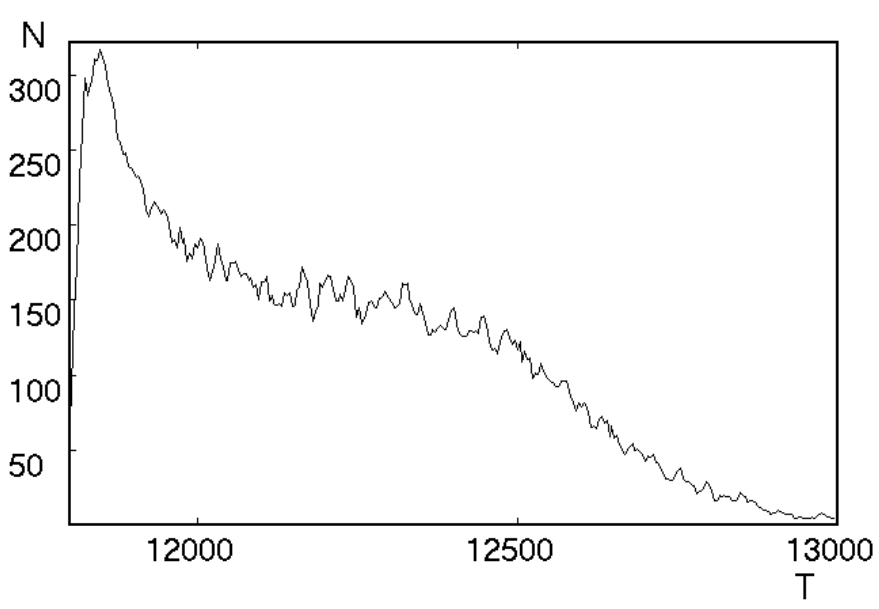

Fig. 5. The temperature distribution of bright $\left(T_{\mathrm{b}}>11800 \mathrm{~K}\right)$ regions at latitudes exceeding $50^{\circ}$.

possible that the plateau (or second smoothed maximum) in Fig. 5 is related the polar faculae at high solar latitudes (see also Figs. 2 and 8). The possibility of such a connection between radio-enhanced regions at 37 and $87 \mathrm{GHz}$ and polar faculae was found by Riehokainen et al. (1998, 2001). We are going to study this in more detail in future investigations.

\subsection{The lifetime}

Another important parameter of the selected regions is their lifetime. To study this we have developed a special procedure in which we check for the existence of a brightness excess in the same physical position on the solar surface over a given interval of time. We take into account the differential rotation of the Sun (Komm et al. 1993). The regions that are situated close to the solar limb were excluded from the analysis due to large errors in position measurements close to the limb. This limits the maximum measurable time scale for high latitude features to approximately 10 days.

The analysis began from the time scale of 10 days. The number of regions with a lifetime exceeding ten days was found. In the next stage we analyzed the existence of bright regions at progressively shorter time periods, excluding longer-lived regions from the analysis. The results are shown in Fig. 6. At high latitudes, the number of the regions drops with increasing lifetime. About $30 \%$ of the regions have a lifetime below one day. The number of regions with a lifetime of seven days is approximately half of those with one day.

The histogram for the low latitude regions is quite different. The obvious reason is the existence of long-lived active regions that can survive more than one solar rotation. Such regions are rare at high latitudes, as Fig. 6 shows. However, even at low latitudes, a significant number of regions have lifetimes less than a week.
$\mathrm{N}$

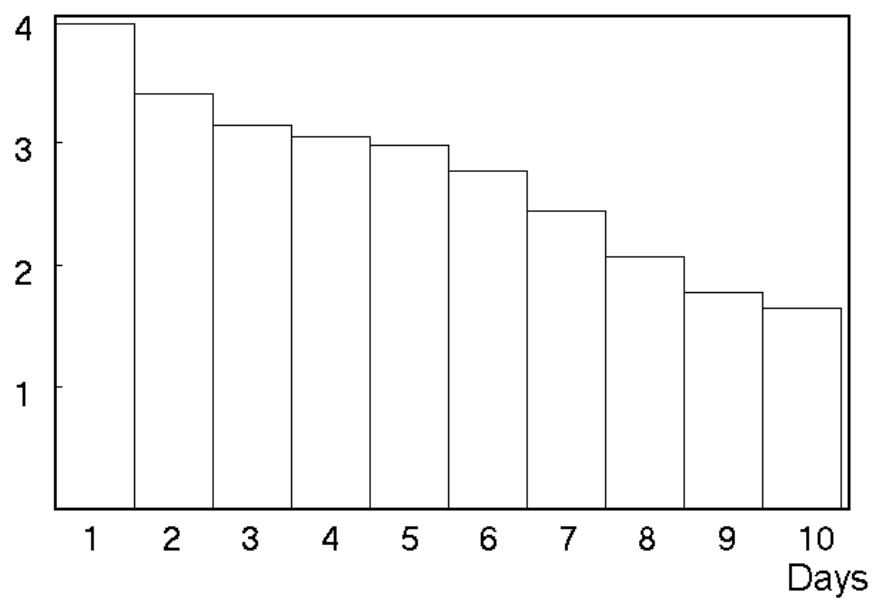

Fig. 6. The histogram of the lifetime distribution of the bright regions at high latitudes. $N$ is the relative number of regions with a given lifetime in days. It can be seen that a sufficient number of radio-bright regions with lifetimes exceeding one day exists for differential rotation determination.

\subsection{Differential rotation}

The existence of a large number of regions living longer than one day allows us to compare their positions on the disk from day to day to find their accurate differential rotation. Figure 7 shows the results of such a study for both bright and dark regions. The bright regions have two different prefered zones for existence, one at low solar latitudes and the second at high solar latitudes (see also Fig. 2). In Fig. 7 (top) this is seen as the alternation of the dark and white areas. Dark regions (bottom) have a more uniform latitude distribution (see Fig. 3), but the density of the distribution is slightly increased around $40^{\circ}-50^{\circ}$. In the low latitude zones the rotation of bright regions can be determined with much higher accuracy than that of the dark regions. The formulae of rotation can be approximated by the functions (for bright and dark regions correspondingly):

$w=13.45-3.0 \sin ^{2} \theta-0.3 \sin ^{4} \theta(\mathrm{deg} /$ day $)$

$w=13.30-1.6 \sin ^{2} \theta-2.0 \sin ^{4} \theta(\mathrm{deg} / \mathrm{day})$.

Data points with a deviation more than 2 (deg/day) from the formula of Komm et al. (1993)

$w=14.38-0.9856-1.95 \sin ^{2} \theta-2.17 \sin ^{4} \theta(\mathrm{deg} / \mathrm{day})$

were excluded from Fig. 7 for simplicity, but were included in the analysis.

A more detailed analysis of differential rotation of the Sun and its variation with the phase of the solar activity will be the subject of a companion paper.

\section{Discussion}

The time interval used in this study (1992-2001) allows us to study the epoch of the polar solar activity during the 


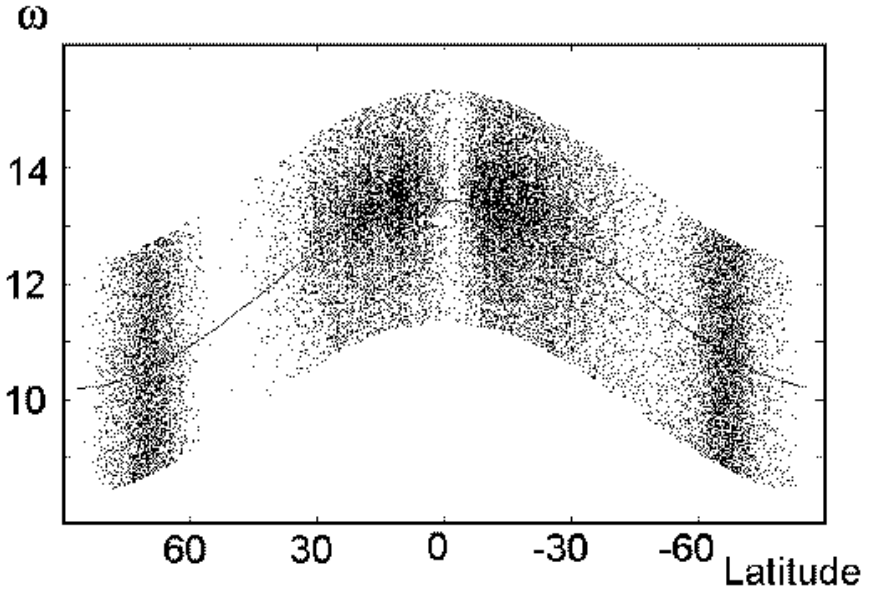

()

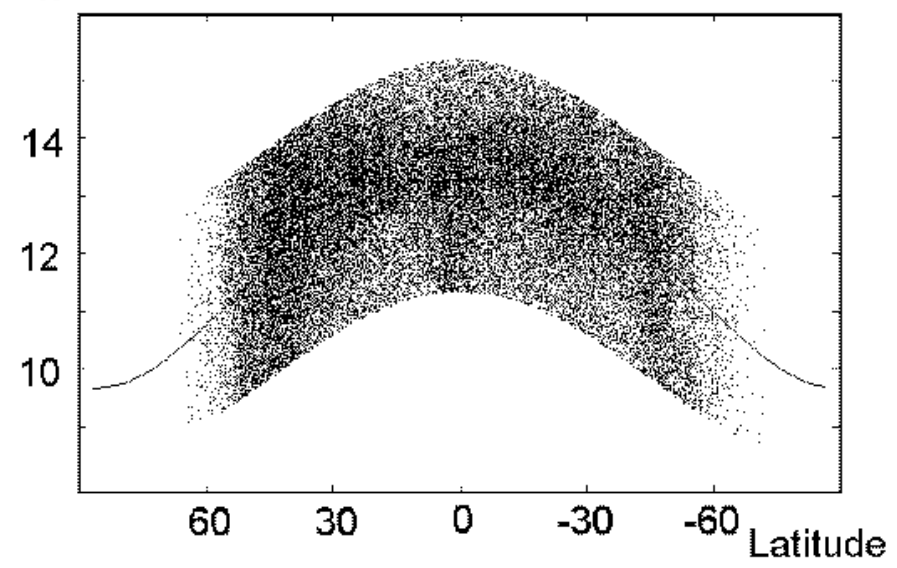

Fig. 7. The latitude dependence of rotational velocity for bright regions (top) and for dark regions (bottom). See text for more explanations.

minimum phase of sunspot activity (between the 22nd and 23rd solar cycles). During this period one usually observes an increase in the number of bright regions at high heliographic latitudes. In our case the maximum was registered at a latitude of about 70 degrees in 1996-1997.

To proceed with a physical interpretation of the results we compare our indices of the Nobeyama radio data with some other types of polar activity. The longest coverage is based on observations of the polar faculae in white light. In Fig. 8 we present their latitude-time distribution, based on observations at the Kislovodsk mountain solar station of the Pulkovo observatory for the period of 1991-2001. In the same figure we also show the distribution of the sunspots and positions of the neutral lines of the solar magnetic fields which mark the border between the two magnetic polarities of the large-scale magnetic field of the Sun. These were determined from the synoptic H-alpha maps made at the Kislovodsk station. A comparison of Figs. 2 and 8 shows that the distribution of bright high latitude radio regions mimics that of the polar faculae. A similar result was obtained by Riehokainen et al. (1998) for the enhanced temperature regions at $37 \mathrm{GHz}$ for the time interval 1982-1995. However, one should keep in mind that
$\Theta$

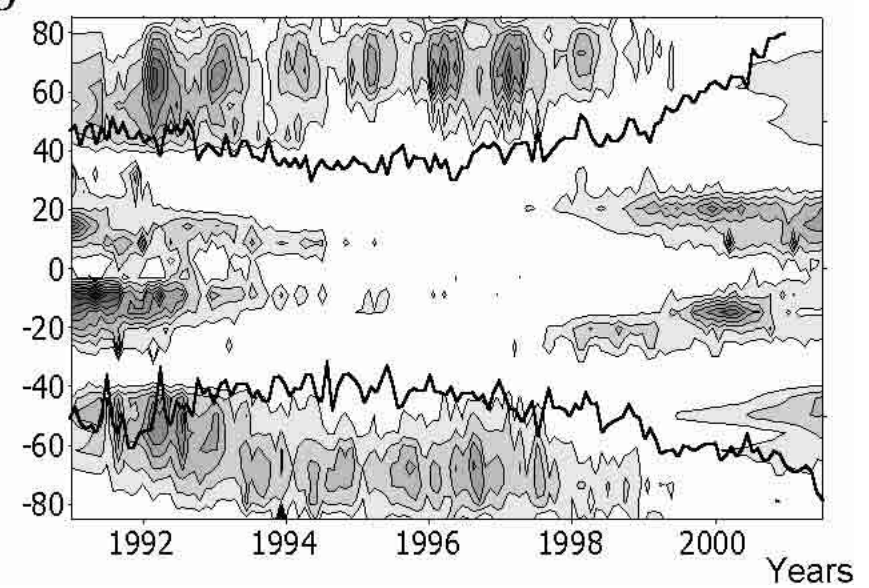

Fig. 8. The polar faculae, the sunspot and the magnetic neutral line cycles according to Kislovodsk observations. The position of the magnetic neutral line is shown by the thick black line. The polar faculae are seen at latitudes above 40 degrees (approximately separated from the sunspots by the magnetic neutral line).

not all polar faculae have counterparts in radio emission (Riehokainen et al. 2001; Gelfreikh et al. 2000).

The high level of similarity between the diagrams for the radio features (Figs. 2 and 3) and for the optical activity (Fig. 8) is strong evidence in favor of a common nature of the observed phenomena. It implies the strengthening of the magnetic fields at high latitudes during the epoch of minimal equatorial solar activity. There is also no reason to conclude that the polar activity is due to the drift of magnetic elements from the lower latitudes of high sunspot activity, as was proposed in a number of previous studies (Makarov \& Makarova 1996). If that were the case, one would expect a strong drift during the period of high polar activity in 1994-1998, which is not observed. Another problem of interest is the difference between magnetic structures of the low and high latitude active microwave regions. The high latitude bright radio regions are superposed on a unipolar background. In contrast, low latitude radio brightenings are superposed on bipolar magnetic structures and the strongest brightenings are usually connected with the positions of the magnetic neutral lines at the photospheric level. The problem of modelling such radio sources, taking into account their magnetic nature, still requires a proper analysis.

Concerning the regions of low radio brightening (dark regions), their latitude-time diagram (Fig. 3) shows the absence of such regions during the minimum of the solar cycle. Their distribution also shows a good agreement with the distribution of magnetic field polarity found from H-alpha maps.

\section{Conclusion}

In this paper we found that during the solar activity cycle the bright radio regions $\left(T_{\mathrm{b}}>11800 \mathrm{~K}\right)$ exhibit both a low latitude component (corresponding to the sunspot 
butterfly diagram) and a high latitude component (corresponding to the polar faculae distribution). The high latitude activity is not simply an extension of the low latitude activity (for example, due to drift of magnetic elements), but instead a separate component of the global solar activity cycle. It was also established that the distribution of the dark radio regions coincides with the zonal structure of the filament belts during the solar cycle. In addition, we analysed some typical characteristics of the radio regions such as their lifetime, area and temperature.

In conclusion, we would like to draw attention to the fact that with the regular two dimensional radio observations of the Sun we have gained a new method to analyze practically all manifestations of the global solar cycle. It is of special value because other methods refer usually to only one or to a few types of activity. The other essential advantage of the radio observations is their regularity with full daily coverage (eight hours per day at Nobeyama since July 1992) with a very weak dependence on weather conditions. In order to proceed in the study of the solar activity cycles, further analysis of the physical nature and modelling of different plasma structures observed at radio waves is highly desirable. The spectral analysis of the radio structures at microwaves is also highly desirable, and would give clues regarding the physics of the observed plasma structures responsible for the radio emission analyzed in this paper. At present such observations are available only in the one dimensional mode with the RATAN-600 (Grebinskij et al. 2000).

Acknowledgements. This work was partially supported by the grants: RFBR 99-02-16171, 99-02-16200, 00-02-16355, and the
National Russian Program "Astronomy" 1.5.4.6. We thank E. Valtaoja for comments on the paper.

\section{References}

Gelfreikh, G. B. 1999, Proc. of the Nobeyama Symp., NRO report, 79,41

Gelfreikh, G. B., Makarov, V. I., \& Tlatov, A. G. 2000, Phys. Chem. Earth (C), 25, No. 5-6, 437

Grebinskij, A., Bogod, V., Gelfreikh, G., Urpo, S., Pohjolainen, S., \& Shibasaki, K. 2000, A\&A, 144, 169

Howard, R., \& LaBonte, B. J. 1980, AJ, 239, L33

Homann, T., Kneer, F., \& Makarov, V. I. 1997, Sol. Phys., 175, 81

Komm, R. W., Howard, R., \& Harvey, J. W. 1993, Sol. Phys., 143, 19

Legrand, J. P., \& Simon, P. A. 1983, J. Geophys. Res., 88, 1

Leroy, J. L., \& Noens, J. C. 1983, A\&A, 120, L1

Makarov, V. I., \& Makarova, V. V. 1996, Sol. Phys., 163, 267

Makarov, V. I., \& Sivaraman, K. R. 1989, Sol. Phys., 119, 35

Makarov, V. I., Tlatov, A. G., Callebaut, D. K., Obridko, V. N., \& Shelting, B. D. 2001, Sol. Phys., 198, 409

Makarov, V. I., Tlatov, A. G., Callebaut, D. K., \& Obridko, V. N. 2002, Sol. Phys., in press

Nakajima, H., Nishio, M., Enome, Sh., et al. 1994, Proc. EEEA, 82,705

Riehokainen, A., Urpo, S., \& Valtaoja, E. 1998, A\&A, 333, 741

Riehokainen, A., Urpo, S., Makarov, V. I., Makarova, V. V., \& Tlatov, A. G. 2001, A\&A, 366, 676

Wilson, P. R., Altrock, R. C., Harvey, K. L., Martin, S. F., \& Snodgrass, H. B. 1988, Nature, 333, 748

Shibasaki, K. 1999, Proc. of the Nobeyama Symp., NRO report, 479,1 